\title{
Identifying model violations under the multispecies coalescent model using P2C2M.SNAPP
}

\author{
Drew J Duckett ${ }^{1}$, Tara A Pelletier $^{2}$, Bryan C Carstens ${ }^{\text {Corresp. } 3}$ \\ ${ }^{1}$ Department of Evolution, Ecology, \& Organismal Biology, The Ohio State University, Columbus, OH, United States \\ 2 Biology Department, Radford University, Radford, VA, United States \\ 3 Department of Evolution, Ecology and Organismal Biology, The Ohio State University, Columbus, OH, United States \\ Corresponding Author: Bryan C Carstens \\ Email address: carstens.12@osu.edu
}

Phylogenetic estimation under the multispecies coalescent model (MSCM) assumes all incongruence among loci is caused by incomplete lineage sorting. Therefore, applying the MSCM to datasets that contain incongruence that is caused by other processes, such as gene flow, can lead to biased phylogeny estimates. To identify possible bias when using the MSCM, we present P2C2M.SNAPP. P2C2M.SNAPP is an R package that identifies model violations using posterior predictive simulation. P2C2M.SNAPP uses the posterior distribution of species trees output by the software package SNAPP to simulate posterior predictive datasets under the MSCM, and then uses summary statistics to compare either the empirical data or the posterior distribution to the posterior predictive distribution to identify model violations. In simulation testing, P2C2M.SNAPP correctly classified up to $83 \%$ of datasets (depending on the summary statistic used) as to whether or not they violated the MSCM model. P2C2M.SNAPP represents a user-friendly way for researchers to perform posterior predictive model checks when using the popular SNAPP phylogenetic estimation program. It is freely available as an $\mathrm{R}$ package, along with additional program details and tutorials. 


\section{Identifying model violations under the Multispecies}

3 Coalescent Model using P2C2M.SNAPP

Drew J. Duckett ${ }^{1}$, Tara A. Pelletier ${ }^{2}$, Bryan C. Carstens ${ }^{1 *}$

${ }^{1}$ Department of Evolution, Ecology, \& Organismal Biology, The Ohio State University,

9 Columbus, $\mathrm{OH}$, United States of America

${ }^{2}$ Biology Department, Radford University, Radford, VA, United States of America

11

12

13

14

Corresponding Author:

Bryan C. Carstens

Department of Evolution, Ecology, \& Organismal Biology, Museum of Biological Diversity, The Ohio State University, 1315 Kinnear Rd., Columbus, OH 43210, United States of America Email address: carstens.12@osu.edu

\section{Abstract}

Phylogenetic estimation under the multispecies coalescent model (MSCM) assumes all incongruence among loci is caused by incomplete lineage sorting. Therefore, applying the MSCM to datasets that contain incongruence that is caused by other processes, such as gene flow, can lead to biased phylogeny estimates. To identify possible bias when using the MSCM, we present P2C2M.SNAPP. P2C2M.SNAPP is an R package that identifies model violations using posterior predictive simulation. P2C2M.SNAPP uses the posterior distribution of species trees output by the software package SNAPP to simulate posterior predictive datasets under the MSCM, and then uses summary statistics to compare either the empirical data or the posterior distribution to the posterior predictive distribution to identify model violations. In simulation testing, P2C2M.SNAPP correctly classified up to $83 \%$ of datasets (depending on the summary statistic used) as to whether or not they violated the MSCM model. P2C2M.SNAPP represents a user-friendly way for researchers to perform posterior predictive model checks when using the popular SNAPP phylogenetic estimation program. It is freely available as an R package, along with additional program details and tutorials. 


\section{Introduction}

40 Alleles that are shared across taxa present a formidable challenge to phylogenetic inference.

41 Species tree inference methods were introduced in an attempt to infer phylogeny without the 42 potentially confounding effects caused by ancestral alleles that were shared across OTUs

43 (Maddison, 1997; Carstens \& Knowles 2007). Since the biological mechanisms that lead to this 44 process (i.e., incomplete lineage sorting) commonly occur at shallow levels of phylogenetic 45 divergence, species trees have largely (but not exclusively; e.g., Prum et al., 2015) been applied 46 near the species boundary, and often in clades where species limits are not entirely clear (Satler, 47 Carstens \& Hedin, 2013). Such applications of the species tree model make the implicit 48 assumption that alleles shared across lineages result from incompletely sorted ancestral 49 polymorphism, even though gene flow is possible in closely related taxa. While gene flow was 50 once considered rare above the species level (at least in animals), recent investigations have 51 suggested that it is more common than previously recognized (e.g., snowshoe hares: MeloFerreira et al. (2014), chipmunks: Sullivan et al. (2014), bears: Kumar et al. (2017), and Myotis bats: Morales et al. (2017)).

54

55

56

57

58

59

60

61

62

63

64

65

66

67

68

69

70

71

72

73

74

75

76

77

78

Given that gene flow has been shown to bias estimates of both topology and branch lengths when it is not accounted for in a phylogenetic analysis (Eckert \& Carstens, 2008; Leache et al., 2013), evolutionary biologists should (at the least) consider the possibility that gene flow has interfered with phylogeny estimation, particularly when inferring phylogeny from closely related species where reproductive isolation may not be complete. One approach is to look for evidence of gene flow in the data, for example by searching for alleles that are shared across non-sister taxa because such alleles are more likely to result from gene flow than coalescent processes. However, this is likely to be a laborious process, particularly in genomic datasets, and gene flow can be easily missed in studies that do not analyze data from all possible hybridization/introgression events. It is considerably more efficient to utilize statistical methods, such as posterior predictive simulation, that seek to determine whether a given dataset violates the model assumptions of the phylogenetic analysis (e.g., Goldman, 1993; Reid et al., 2014).

Posterior predictive approaches have been developed for several types of phylogenetic models, including models of sequence evolution (Huelsenbeck et al., 2001; Brown, 2014b), species delimitation (Barley \& Thomson, 2016; Barley, Brown \& Thomson, 2018), and species tree estimation (Reid et al., 2014). The basic approach is to (i) draw parameter values from the posterior distribution, (ii) simulate new datasets using these parameter values under the model assumed by the analysis, (iii) analyze the simulated data to generate posterior predictive distributions, and (iv) calculate and compare summary statistics from either the empirical data or the posterior distribution to the posterior predictive distribution. Analytical models that represent a good fit for the empirical data should produce summary statistics values that fall within the distribution of values estimated under the correct model with posterior predictive datasets (Brown, 2014b). Recently, posterior predictive checks have been incorporated into an R package 
(Posterior Predictive Checks of Coalescent Models (P2C2M): Gruenstaeudl et al., 2016) for the MSCM framework. $\mathrm{P} 2 \mathrm{C} 2 \mathrm{M}$ was designed to easily allow users to perform posterior predictive analyses, but the program uses the species tree inference package *BEAST which is intended for smaller, sub-genomic data sets (Heled \& Drummond, 2009). Here, we expand P2C2M to the genomic era so that it can be used to conduct posterior predictive checks using single nucleotide polymorphisms (SNPs) in the SNAPP implementation of the MSCM (Bryant et al., 2012).

\section{Materials \& Methods}

\section{Pipeline}

The posterior predictive simulation framework for SNAPP (P2C2M.SNAPP) has been implemented as an R package ( $R$ Core Team, 2018), with detailed program settings described in the package documentation and tutorial. P2C2M.SNAPP differs from the original P2C2M in the input datatype (sequence data in the original versus SNP data in the SNAPP version) and consequently the summary statistics used to compare empirical and posterior predictive datasets. User input to P2C2M.SNAPP includes the SNAPP .xml formatted input file, the posterior distribution of species trees and log file from a SNAPP analysis, and a metadata text file containing the number of SNPs used, an estimated mutation rate, and the number of samples per group. Importantly, P2C2M.SNAPP assumes users have properly performed SNAPP species tree estimation analysis, including selecting the proper priors for their data and study system and checking for Markov chain convergence. Because P2C2M.SNAPP relies on the posterior distribution of species trees, users should retain at least 100 trees in the posterior distribution to sample from. P2C2M.SNAPP proceeds as follows: (i) it samples, either uniformly or at random, a user-specified number of species trees from the posterior distribution, (ii) extracts taxonomic relationships and branch lengths from each tree, and (iii) for each tree sampled from the posterior, it simulates a posterior predictive dataset under the MSCM using fastsimcoal2, a userspecified number of simulations (Excoffier et al., 2013) and the parameters extracted from the metadata text file (Figure 1). Posterior predictive datasets are converted to SNAPP .xml files, and users conduct SNAPP analyses on each posterior predictive dataset using the .xml file output by P2C2M.SNAPP. Prior distributions and Markov chain parameters for the posterior predictive SNAPP analyses are recycled from those used in the original SNAPP analysis in order to maintain consistency. Given the intense computational requirements of SNAPP, generation of the posterior predictive species tree distributions is best conducted using parallel computation. Example scripts for automating SNAPP analyses are included with the tutorial (http://www.github.com/P2C2M/P2C2M_SNAPP). The results of SNAPP analyses on the posterior predictive datasets (i.e., SNAPP .xml files, posterior species tree distributions, and log files) are subsequently used as input for the second stage of the P2C2M.SNAPP analysis, where summary statistics from the posterior and posterior predictive datasets are calculated and compared to identify model violations.

\section{Summary Statistics}


119 Generally, summary statistics used in posterior predictive checks fall into two categories: data120 based, which compare the empirical and posterior predictive datasets themselves, and inference121 based, which compare the inferences produced by analyzing the empirical and posterior

122

123

124

125

126

127

128

129

130

131

132

133

134

135

136

137

138

139

140

141

142

143

144

145

146

147

148

149

150

151

152

153

154

155

156

157

158

predictive datasets (Brown, 2014a; Barley and Thomson, 2016). Inference-based statistics can provide more insight as to whether a model violation affects the end result (e.g., the estimated species tree), but can also be more computationally difficult because posterior predictive datasets need to be analyzed with the same methods as the posterior (i.e., species trees need to be estimated with SNAPP). In contrast, data-based statistics do not determine the effect a model violation has on the inference, but are usually computationally efficient. Both data-based and inference-based summary statistics were evaluated to determine which statistic identified model violations to the MSCM with the highest accuracy. Data-based statistics included several based on a fixation index $\left(\mathrm{F}_{\mathrm{ST}}\right)$, and inference-based statistics included tree metrics based on RobinsonFoulds or Kuhner-Felsenstein tree distances, and the mean and standard deviation of tree likelihoods. $\mathrm{F}_{\mathrm{ST}}$ is a commonly used metric for measuring the amount of population structure, and the value ranges from 0 to 1 , with populations becoming more structured as $\mathrm{F}_{\mathrm{ST}}$ approaches 1 (Wright, 1949). Therefore, lineages exchanging genes should exhibit lower $\mathrm{F}_{\mathrm{ST}}$ values because they will share alleles. Pairwise $\mathrm{F}_{\mathrm{ST}}$ was calculated across all loci in the KRIS package (Chaichoompu, 2018). $\mathrm{F}_{\mathrm{ST}}$ summary statistics included mean $\mathrm{F}_{\mathrm{ST}}$, range of $\mathrm{F}_{\mathrm{ST}}$, and an $\mathrm{F}_{\mathrm{ST}}$ outlier test. For the mean and range $\mathrm{F}_{\mathrm{ST}}$ statistics, the summaries are calculated for each posterior predictive dataset and the empirical dataset. Similar to a two-tailed posterior predictive $p$-value (Brown, 2014a; Barley, Brown \& Thomson, 2018), a $p$-value is calculated by counting the number of posterior predictive datasets with summary statistic values falling above and below the empirical value, multiplying the lesser of these values by two (to emulate a two-tail test), and then dividing by the total number of posterior predictive datasets. We consider $p$-values less than $\alpha=0.05$ to indicate a model violation. The $\mathrm{F}_{\mathrm{ST}}$ outlier test was conducted by calculating the average difference between empirical and simulated values for each pairwise comparison, and then conducting an outlier test using the function boxplot.stats in the grDevices package ( $R$ core team, 2018). Since we consider any detected outlier to indicate a model violation, the pairwise outliers identified by this approach can be used to identify lineages exchanging genes.

Two tree distance metrics were also examined, one that considers topology only and one that considers topology and branch lengths. The Robinson-Foulds distance compares the topology between two phylogenetic trees, with values ranging from 0 (no topology difference) to 1 (completely different topologies) (Robinson \& Foulds, 1981). High rates of gene flow can influence topology estimation and result in an errant clade consisting of two lineages that are not closely related but that share alleles due to gene flow. However, it may be more likely that gene flow may mislead the estimation of branch lengths even if the underlying topology is correct. Therefore, a tree distance metric incorporating branch length differences as well as topology may prove to be a useful summary statistic for comparing empirical and posterior predictive datasets. One such metric is the Kuhner-Felsenstein distance, which also calculates values between 0 (no

Peer) reviewing PDF | (2019:04:36665:1:1:NEW 30 Sep 2019) 
159

160

161

162

163

164

165

166

167

168

169

170

171

172

173

174

175

176

177

178

179

180

181

182

183

184

185

186

187

188

189

190

191

192

193

194

195

196

197

198

difference between trees) and 1 (high difference between trees) (Kuhner \& Felsenstein, 1994). Both tree distance metrics were calculated using the ape package (Paradis, Claude \& Strimmer, 2018). If posterior trees were estimated from a dataset that violates the MSCM model, we expect that these trees will have large tree distances when compared to posterior predictive trees simulated under the correct model (MSCM). Additionally, as all posterior trees reflect similar processes in the empirical dataset, we expect that tree distances among trees in the posterior under a model violation will be less than distances between the posterior and posterior predictive trees. Therefore, for the tree distance metrics, 1000 comparisons were performed between random trees sampled from the original SNAPP posterior distribution of species trees to create a null distribution. Then 100 random trees from the posterior predictive distribution were compared to the posterior tree they were simulated from, and this was repeated for each posterior predictive dataset. A $p$-value was calculated by counting the number of posterior predictive to posterior tree comparisons falling above the $95 \%$ null distribution (values below the $95 \%$ null distribution represent high similarity between posterior and posterior predictive datasets, and thus are not useful for detecting violations), and then dividing by the total number of comparisons. We consider $p$-values greater than $\alpha=0.05$ to indicate model violations. Finally, because it is likely more difficult to estimate trees with high probability under an incorrect model, we examined the mean and standard deviation of tree likelihoods as calculated from SNAPP output. The evaluation of the likelihood statistics follows that of the-mean and range $\mathrm{F}_{\mathrm{ST}}$ statistics, described above.

\section{Testing}

P2C2M.SNAPP was tested by simulating data under the MSCM and via a second simulation under the MSCM with gene flow (i.e., MSCM+m) (Figure 2). One hundred replicates were performed under each model. Note that the MSCM $+m$ model is a clear violation of the underlying coalescent model that is incorporated into SNAPP because an appreciable portion of the shared polymorphism results from gene flow. All simulations were based on 2000 SNPs, 6 species with two individuals sampled per species, an effective population size $\left(\mathrm{N}_{\mathrm{e}}\right)$ of $100 \mathrm{~K}$ individuals, and a symmetric topology with speciation event times of $5 \mathrm{~N}, 10 \mathrm{~N}$, and $20 \mathrm{~N}$ generations. The number of SNPs simulated is lower than many empirical data sets, but it allows SNAPP analyses to proceed in less time and should represent a conservative test of the ability of P2C2M.SNAPP to detect model violations because the performance of SNAPP generally improves with additional data (Bryant et al. 2012). The MSCM $+m$ model was designed as a secondary contact scenario, with gene flow between two lineages starting at $2.5 \mathrm{~N}$ generations in the past and continuing until the present. Both the species experiencing gene flow and the rate of gene flow were selected at random, with the rate of gene flow having a uniform prior distribution between 0.5 and 5 migrants per generation. Simulations were performed using fastsimcoal 2 (Excoffier et al., 2013) and simulated datasets were converted to SNAPP .xml files using custom Python scripts (http://www.github.com/P2C2M/P2C2M_SNAPP). SNAPP analyses were conducted using the following parameters: a gamma prior on the rate of species divergence

Peer] reviewing PDF | (2019:04:36665:1:1:NEW 30 Sep 2019) 
199

200

201

202

203

204

205

206

207

208

209

210

211

212

213

214

215

216

217

218

219

220

221

222

223

224

225

226

227

228

229

230

231

232

233

234

235

236

237

238

(lambda) under the Yule speciation prior with $\alpha=2$ and $\beta=200$, a gamma rate prior on ancestral effective population sizes (theta), mutation rate of $\mu=\nu=1.0$, and a Markov chain of $1 \mathrm{M}$ steps with $100 \mathrm{~K}$ burn-in steps and sampling every $1 \mathrm{~K}$ steps. In order to evaluate the summary statistics, the number of correct inferences, false positives, and false negatives were calculated for each model (200 total) using the posterior and posterior predictive distributions from the SNAPP analyses. False positives are defined as datasets simulated under the MSCM that were indicated as model violations by P2C2M.SNAPP. Conversely, false negatives are defined as datasets simulated under the MSCM $+m$ model that were not detected as model violations by P2C2M.SNAPP. Mathews Correlation Coefficient (MCC; Mathews, 1975) was also calculated for each summary statistic with the R package mltools (Gorman, 2018). The MCC takes into account false negatives and positives while measuring how well a binary classifier performs, in this case whether a summary statistic correctly classifies a dataset. The coefficient ranges from 1 to 1 with -1 indicating the classifier is completely wrong and 1 indicating it is completely correct. Additionally, pairwise $\mathrm{F}_{\mathrm{ST}}$ outliers were compared to the $\mathrm{MSCM}+m$ simulation parameters to assess if the statistic could identify the species exchanging genes to cause model violations. Finally, $p$-values for each simulation were plotted against gene flow to identify any trends between the level of gene flow and summary statistic performance.

\section{Results}

P2C2M.SNAPP requires about five minutes on an average laptop (2.6GHz Intel Core i5, 8GB RAM) to generate posterior predictive datasets at the beginning of the pipeline and to evaluate summary statistics in order to identify model violations at the end of the pipeline. However, the entire pipeline requires a considerable amount of time due to the demands of the SNAPP program itself. For example, each replicate of our simulation testing required 300-450 CPU hours on the Pitzer cluster (28 cores and 112GB RAM) at the Ohio Supercomputer Center (Ohio Supercomputer Center, 2018). While this is clearly not an analysis that users would likely conduct on a laptop computer, the time required for users to analyze their data using P2C2M.SNAPP is still likely to be less than the time required to collect the samples, generate the sequencing libraries, and conduct the bioinformatics.

There was a dramatic difference across summary statistics in the ability of P2C2M.SNAPP to identify model violations due to gene flow (Table 1). The mean and range of pairwise $\mathrm{F}_{\mathrm{ST}}$ values correctly classified datasets in only $33 \%$ and $41 \%$ of simulations respectively (MCC equals -0.45 and -0.32 respectively; Figure 2). Each of these statistics exhibited a large number of false positives in which a model violation was detected in a dataset that was simulated under the assumptions of the MSCM. While the pairwise $\mathrm{F}_{\mathrm{ST}}$ outlier test classified $46 \%$ of datasets correctly, the majority of misclassifications were false negatives (MCC equals -0.17). Additionally, we examined the ability of the pairwise $\mathrm{F}_{\mathrm{ST}}$ outlier test to identify the OTUs exchanging genes in the MSCM $+m$ datasets. As the statistic only identified $3 \%$ of true model violations, there were very few datasets to test. The pairwise $\mathrm{F}_{\mathrm{ST}}$ outlier test did not correctly 
239 identify the OTUs exchanging genes in any of the datasets. Each tree statistic correctly classified

240 only around half of the datasets, with a high number of false negatives when using the Robinson-

241 Foulds distance and a similar number of false positives when using the Kuhner-Felsenstein

242 distance (MCC equals 0 for each). Similarly, evaluations by the mean tree likelihood statistic

243 were split evenly between correct inferences and false positives (MCC equals -0.29). Our results

244 identified one statistic that performed well. The standard deviation of tree likelihoods correctly

245 classified $83 \%$ of simulated datasets, with $14 \%$ false negatives and $3 \%$ false positives (MCC

246 equals 0.68 ). Only two summary statistics showed a trend between the rate of gene flow and the

$247 p$-value of posterior predictive checks (Figure 3). For the range of pairwise $\mathrm{F}_{\mathrm{ST}}$ and mean of tree

248 likelihoods, $p$-values decreased as the rate of gene flow increased.

249

250

\section{Discussion}

251

252

253

254

255

256

257

258

259

260

261

262

263

264

265

266

267

268

269

270

271

272

273

274

275

276

277

278

While it has been known for some time that model violations can degrade the accuracy of phylogenetic estimation (Huelsenbeck et al., 2001; Eckert \& Carstens 2008, Leache et al., 2013; Brown, 2014a; Reid et al., 2014; Barley \& Thomson, 2016; Barley, Brown \& Thomson, 2018), few studies explore possible violations inherent in their datasets to the phylogenetic model used in the analysis (e.g. Morales et al., 2017; Diaz et al., 2018; Richards et al., 2018). Apart from the computational demands of the SNAPP analyses, P2C2M.SNAPP represents a user-friendly and reasonably accurate method for identifying violations of the MSCM. The package and tutorial, including examples for running analyses, are available on the $\mathrm{P} 2 \mathrm{C} 2 \mathrm{M}$ Github page (https://github.com/P2C2M/P2C2M_SNAPP).

Our simulation testing indicates that the standard deviation of tree likelihoods is useful in identifying datasets that contain SNP patterns resulting from gene flow between lineages, a clear violation of SNAPP's analytical model. This statistic is likely useful because datasets that violate the MSCM model will be more difficult to estimate and may exhibit posterior distributions with poor convergence. Methods examining the variance within and between posterior and posterior predictive datasets have previously proven useful for posterior predictive checks of Bayesian phylogenetic models (Gelfand and Ghosh, 1998; Lewis et al., 2013). Users of P2C2M.SNAPP should focus on the standard deviation of tree likelihoods when assessing their datasets.

Although higher rates of gene flow should result in a more egregious model violation, it does not appear to be the case that model violations are easier to detect under scenarios with high rates of gene flow. Two summary statistics (range of pairwise $\mathrm{F}_{\mathrm{ST}}$, mean of tree likelihoods) exhibit an inverse correlation between gene flow and the resulting $p$-value, but both exhibited a high rate of false positives which makes them a poor choice for use in posterior predictive checks. While this relationship does not hold for the standard deviation of tree likelihoods, the statistic is able to detect model violations equally well across a range of migration rates.

Several statistics were much less useful than we expected them to be. Although the tree distance metrics are conceptually simple, their poor performance may be explained by the reliance of

Peer) reviewing PDF | (2019:04:36665:1:1:NEW 30 Sep 2019) 
279 posterior predictive simulation on the empirical phylogeny. Because the posterior predictive data 280 sets are simulated from the empirical phylogeny estimates, inaccuracies in topology and branch 281 lengths of the empirical phylogeny due to gene flow are translated into inaccurate topology and 282 divergence times in the posterior predictive simulations. The result is similar, but inaccurate 283 phylogeny estimates for each data type. $\mathrm{F}_{\mathrm{ST}}$ is a popular metric in population genetics for 284 examining population structure and gene flow, but may not be applicable to phylogenetic

285

286

287

288

289

290

291

292

293

294

295

296

297

298

299

300

301

302

303

304

305

306

307

308

309

310

311

312

313

314

315

316

317

318 analyses due to fixed differences among lineages. It is possible that including more samples per lineage may increase the usefulness of $\mathrm{F}_{\mathrm{ST}}$ because more shared polymorphism may be evident, but this may be unfeasible due to the computational requirements of SNAPP. Summary statistics such as $\mathrm{F}_{\mathrm{ST}}$ are appealing because they can be computed from the posterior predictive datasets without additional SNAPP runs, but many existing statistics were developed for population genetic applications. Summary statistics such as the number of shared or private alleles may be useful-Additionally, the calculation of effect sizes could be beneficial to users because it provides information regarding the degree to which model violation has influenced their results Brown, 2014a). Our simulation design investigated a relatively recent diversification scenario because the presence of gene flow is likely to occur when lineages have not become completely reproductively isolated. However, if gene flow occurs in older systems, it should presumably be easier to differentiate from incompletely sorted ancestral polymorphism and thus more easily recognized. Finally, other processes, such as natural selection, also violate the MSCM model, and these additional model violations may also potentially be detectable using the posterior predictive framework implemented in P2C2M.SNAPP, but further research is necessary to identify summary statistics that can detect these violations.

While the detection of a model violation can have implications for the interpretation of a phylogeny estimate, a model violation does not render the data useless. Minimally, researchers should acknowledge the model violation and temper their interpretation of the patterns evident in the phylogeny. Specifically, the possibility that a model violation may have confounded topology estimates or, more likely, biased branch length/divergence time estimates should be addressed. More preferably, researchers should conduct additional analyses to examine the cause of the model violation, as such violations indicate interesting evolutionary processes not accounted for by the MSCM model. In the case of gene flow, model violations can indicate unknown hybridization among OTUs, and lead to the collection of population-level data that can be analyzed using methods such as Migrate- $n$ (Beerli \& Felsenstein, 2001) or Bayesass (Wilson \& Rannala, 2003). Finally, many recently developed models attempt to infer gene flow and phylogeny under the MSCM for small numbers of lineages (e.g. IMa3; Hey et al., 2018, PhyloNet; Wen et al., 2016, SpeciesNetwork; Zhang et al., 2018). Model violations identified by P2C2M.SNAPP are likely to point researchers to additional analyses that will enable them to understand the history of their focal system.

\section{Conclusions}


319 Here we present a new R package for assessing model violations in the species tree estimation

320

321

322

323

324

325

326

327

328

329

330

331

332

333

334

335

336

337

338

339

340

341

342

343

344

345

346

347

348

349

350

351

352

353

354

355

356

357

358

359

360

program SNAPP. The package uses posterior predictive simulations to identify model violations, and is successful in testing with simulated datasets. P2C2M.SNAPP is the newest addition to a small suite of user-friendly programs for conducting posterior predictive checks (Gruenstaeudl et al., 2016). Due to the proven benefit of model checking for phylogenetic analyses, we recommend researchers make posterior predictive checks a routine step in estimating phylogenies.

\section{Acknowledgements}

We would like to thank the Carstens laboratory for constructive conversations about this project and providing feedback on the manuscript before submission. We also thank Remco Bouckaert for answering questions about the SNAPP program and the Ohio Supercomputer Center for early access to the Pitzer computing cluster.

\section{References}

Barley, A. J., \& Thomson, R. C. (2016). Assessing the performance of DNA barcoding using posterior predictive simulations. Molecular Ecology, 25(9), 1944-1957.

Barley, A. J., Brown, J. M., \& Thomson, R. C. (2018). Impact of model violations on the inference of species boundaries under the multispecies coalescent. Systematic Biology, 67(2), 269-284.

Beerli, P., \& Felsenstein, J. (2001). Maximum likelihood estimation of a migration matrix and effective population sizes in $\mathrm{n}$ subpopulations by using a coalescent approach. Proceedings of the National Academy of Sciences, 98(8), 4563-4568.

Brown, J. M. (2014a). Detection of implausible phylogenetic inferences using posterior predictive assessment of model fit. Systematic Biology, 63(3), 334-348.

Brown, J. M. (2014b). Predictive approaches to assessing the fit of evolutionary models. Systematic biology, 63(3), 289-292.

Bryant, D., Bouckaert, R., Felsenstein, J., Rosenberg, N. A., \& RoyChoudhury, A. (2012). Inferring species trees directly from biallelic genetic markers: bypassing gene trees in a full coalescent analysis. Molecular Biology and Evolution, 29(8), 1917-1932.

Carstens B. C., \& Knowles L. L. (2007). Estimating phylogeny from gene tree probabilities in Melanoplus grasshoppers despite incomplete lineage sorting. Systematic Biology 56, 400411.

Chaichoompu, K., Abegaz, F., Tongsima, S., Shaw, P. J., Sakuntabhai, A., Pereira, L., \& Van Steen, K. (2018). KRIS: Keen and Reliable Interface Subroutines for Bioinformatic Analysis. R package version 1.1.1. Available at: https://CRAN.R-project.org/package=KRIS.

Diaz, F., Lima, A. L. A., Nakamura, A. M., Fernandes, F., Sobrinho Jr, I., \& De Brito, R. A. (2018). Evidence for introgression among three species of the Anastrepha fraterculus group, a radiating species complex of fruit flies. Frontiers in genetics, 9, 359.

Eckert, A. J., \& Carstens, B. C. (2008). Does gene flow destroy phylogenetic signal? The performance of three methods for estimating species phylogenies in the presence of gene flow. Molecular Phylogenetics and Evolution, 49(3), 832-842.

Peer) reviewing PDF | (2019:04:36665:1:1:NEW 30 Sep 2019) 
361 Excoffier, L., Dupanloup, I., Huerta-Sanchez, E., Sousa, V. C., \& Foll, M. (2013). Robust

362

363

364

365

366

367

368

369

370

371

372

373

374

375

376

377

378

379

380

381

382

383

384

385

386

387

388

389

390

391

392

393

394

395

396

397

398

399

400

401 demographic inference from genomic and SNP data. PLoS Genetics, 9(10), e1003905.

Gelfand, A. E., \& Ghosh, S. K. (1998). Model choice: a minimum posterior predictive loss approach. Biometrika, 85(1), 1-11.

Goldman, N. (1993). Statistical tests of models of DNA substitution. Journal of Molecular Evolution, 36(2), 182-198.

Gorman, B. (2018). mltools: Machine Learning Tools. R package version 0.3.5. https://CRAN.Rproject.org/package $=$ mltools.

Gruenstaeudl, M., Reid, N. M., Wheeler, G. L., \& Carstens, B. C. (2016). Posterior predictive checks of coalescent models: P2C2M, an R package. Molecular Ecology Resources, 16(1), 193-205.

Heled, J., \& Drummond, A. J. (2009). Bayesian inference of species trees from multilocus data. Molecular biology and evolution, 27(3), 570-580.

Hey, J., Chung, Y., Sethuraman, A., Lachance, J., Tishkoff, S., Sousa, V. C., \& Wang, Y. (2018). Phylogeny Estimation by Integration over Isolation with Migration Models. Molecular biology and evolution, 35(11), 2805-2818.

Huelsenbeck, J. P., Ronquist, F., Nielsen, R., \& Bollback, J. P. (2001). Bayesian inference of phylogeny and its impact on evolutionary biology. Science, 294(5550), 2310-2314.

Leaché, A. D., Harris, R. B., Rannala, B., \& Yang, Z. (2013). The influence of gene flow on species tree estimation: a simulation study. Systematic Biology, 63(1), 17-30.

Lewis, P. O., Xie, W., Chen, M. H., Fan, Y., \& Kuo, L. (2013). Posterior predictive Bayesian phylogenetic model selection. Systematic biology, 63(3), 309-321.

Kuhner, M. K., \& Felsenstein, J. (1994). A simulation comparison of phylogeny algorithms under equal and unequal evolutionary rates. Molecular biology and evolution, 11(3), 459468.

Kumar, V., Lammers, F., Bidon, T., Pfenninger, M., Kolter, L., Nilsson, M. A., \& Janke, A. (2017). The evolutionary history of bears is characterized by gene flow across species. Scientific reports, 7, 46487.

Maddison, W. P. (1997). Gene trees in species trees. Systematic biology, 46(3), 523-536.

Matthews, B. W. (1975). Comparison of the predicted and observed secondary structure of T4 phage lysozyme. Biochimica et Biophysica Acta (BBA)-Protein Structure, 405(2), 442-451.

Melo-Ferreira, J., Seixas, F. A., Cheng, E., Mills, L. S., \& Alves, P. C. (2014). The hidden history of the snowshoe hare, Lepus americanus: extensive mitochondrial DNA introgression inferred from multilocus genetic variation. Molecular ecology, 23(18), 4617-4630.

Morales, A. E., Jackson, N. D., Dewey, T. A., O’Meara, B. C., \& Carstens, B. C. (2017). Speciation with gene flow in North American Myotis bats. Systematic Biology, 66(3), 440452.

Ohio Supercomputer Center. (2018). Pitzer Supercomputer. Columbus, OH: Ohio Supercomputer Center.

Paradis, E., Claude, J., \& Strimmer, K. (2004). APE: analyses of phylogenetics and evolution in R language. Bioinformatics, 20(2), 289-290.

Peer] reviewing PDF | (2019:04:36665:1:1:NEW 30 Sep 2019) 
402 Prum, R. O., Berv, J. S., Dornburg, A., Field, D. J., Townsend, J. P., Lemmon, E. M., \& 403 Lemmon, A. R. (2015). A comprehensive phylogeny of birds (Aves) using targeted next404 generation DNA sequencing. Nature, 526(7574), 569.

405 R Core Team. (2018). R: A Language and Environment for Statistical Computing. (R Foundation 406 for Statistical Computing). Vienna, Austria. Availabe at http://www.r-project.org. 407 Reid, N. M., Hird, S. M., Brown, J. M., Pelletier, T. A., McVay, J. D., Satler, J. D., \& Carstens, 408 B. C. (2013). Poor fit to the multispecies coalescent is widely detectable in empirical 409 data. Systematic Biology, 63(3), 322-333.

410 Richards, E. J., Brown, J. M., Barley, A. J., Chong, R. A., \& Thomson, R. C. (2018). Variation 411 across mitochondrial gene trees provides evidence for systematic error: How much gene tree $412 \quad$ variation is biological?. Systematic biology, 67(5), 847-860.

413 Robinson, D. F., \& Foulds, L. R. (1981). Comparison of phylogenetic trees. Mathematical 414 biosciences, 53(1-2), 131-147.

415 Satler J. D., Carstens B. C., Hedin M. (2013). Multilocus species delimitation in a complex of 416 morphologically conserved trapdoor spiders (Mygalomorphae, Antrodiaetidae, Aliatypus). 417 Systematic Biology 62, 805-823.

418 Sullivan, J., Demboski, J. R., Bell, K. C., Hird, S., Sarver, B., Reid, N., \& Good, J. M. (2014). 419 Divergence with gene flow within the recent chipmunk radiation (Tamias). Heredity, 113(3), 420185.

421 Wen, D., Yu, Y., Zhu, J., \& Nakhleh, L. (2018). Inferring phylogenetic networks using 422 PhyloNet. Systematic biology, 67(4), 735-740.

423 Wilson, G. A., \& Rannala, B. (2003). Bayesian inference of recent migration rates using 424 multilocus genotypes. Genetics, 163(3), 1177-1191.

425 Wright, S. (1949). The genetical structure of populations. Annals of eugenics, 15(1), 323-354.

426 Zhang, C., Ogilvie, H. A., Drummond, A. J., \& Stadler, T. (2017). Bayesian inference of species 427 networks from multilocus sequence data. Molecular biology and evolution, 35(2), 504-517. 
Figure 1

Workflow of the P2C2M.SNAPP pipeline.

Blue arrows represent the path of the data. Steps outlined in blue are those performed by the user and steps outlined in red are performed by P2C2M.SNAPP. Workflow proceeds from the top of the figure. 


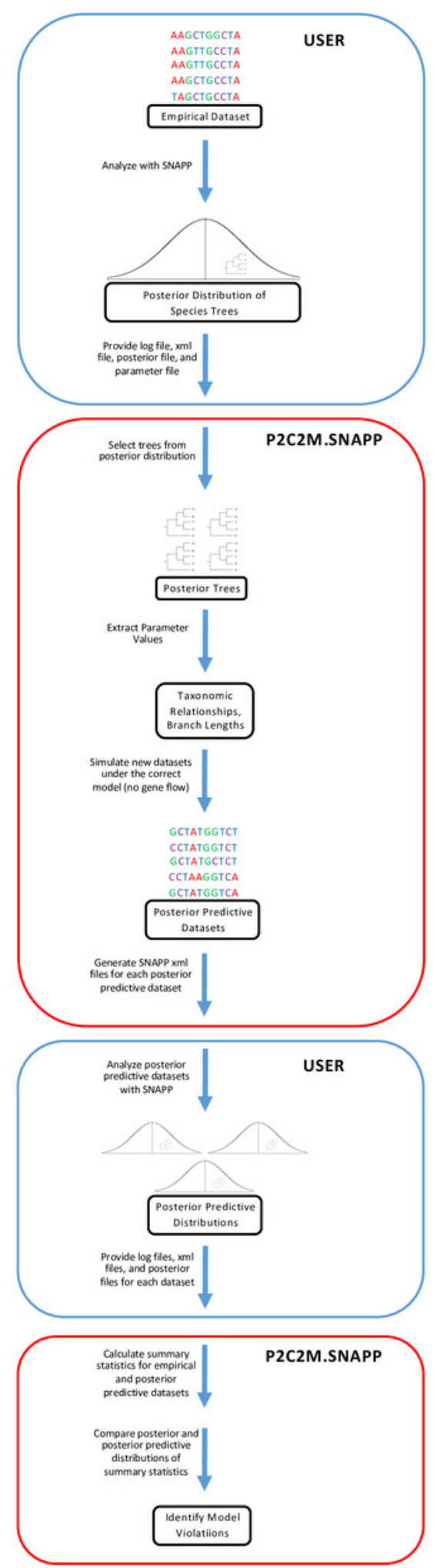


Figure 2

Models used in simulation testing.

A) MSCM model used for simulation testing. B) Example of the MSCM $+m$ model that includes gene flow violating the MSCM model implemented in SNAPP. The amount of gene flow and taxa exchanging genes were randomly selected for each simulation replicate. 

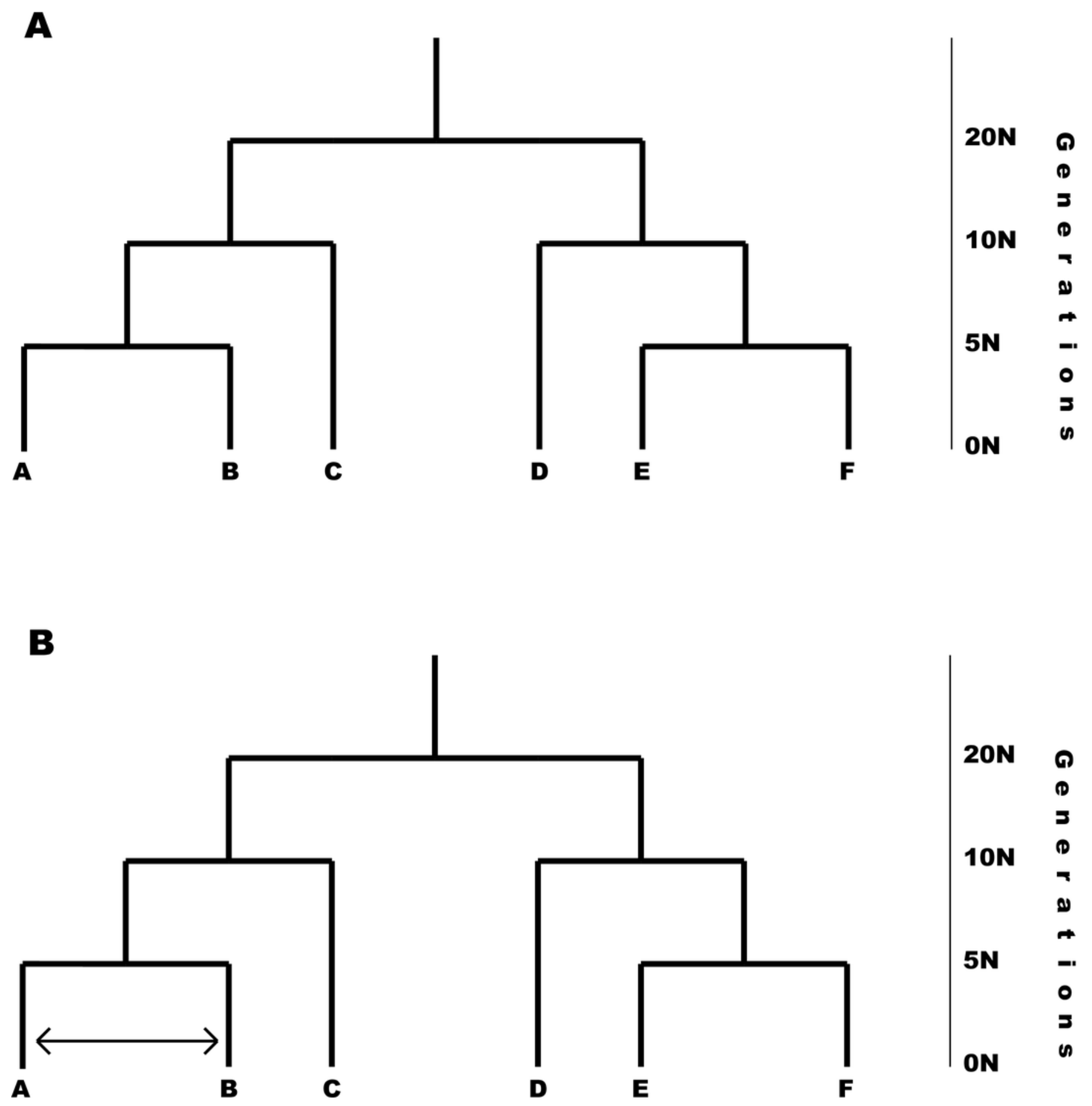


\section{Figure 3}

Correlations between the level of gene flow and the ability of each summary statistic to identify model violations.

The $p$-value for each MSCM $+m$ simulation is plotted against the amount of gene flow simulated with that dataset. FSTA: average pairwise $F_{S T}$, FSTR: range of pairwise $F_{S T}, K F$ : Kuhner-Felsenstein distance, MLM: Mean of the maximum likelihood of posterior trees, MLSD: standard deviation of the maximum likelihood of posterior trees, RF: Robinson-Foulds distance. 

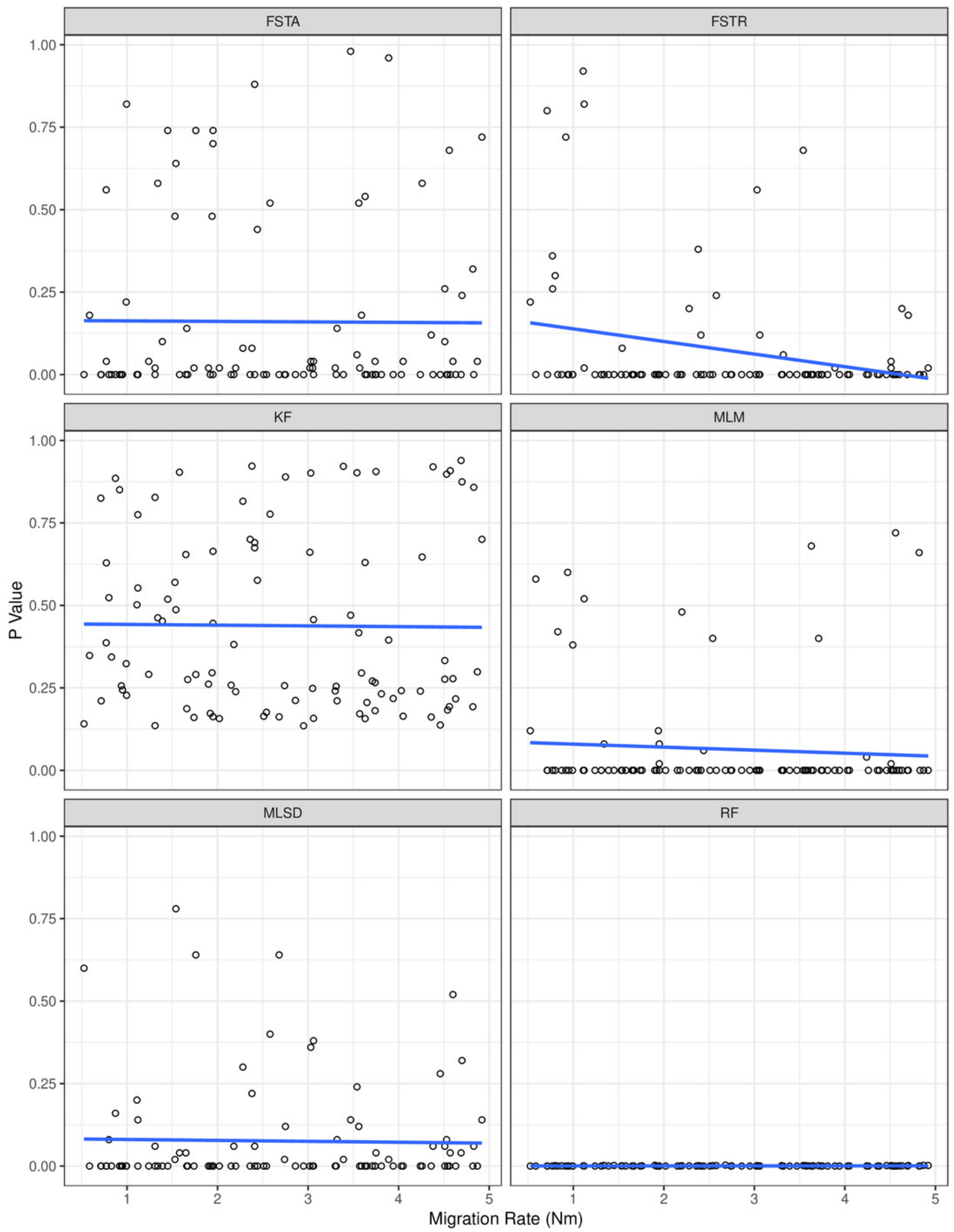


\section{Table $\mathbf{1}$ (on next page)}

Results of Simulation Testing.

Results include all simulations with both the MSCM and MSCM $+m$ models. False positives are datasets simulated under the MSCM model which P2C2M.SNAPP classified as a model violation. False negatives are datatsets simulated under the $\mathrm{MSCM}+m$ model that P2C2M.SNAPP classified as not violating the model implemented in SNAPP. 


\begin{tabular}{|c|c|c|c|c|c|}
\hline Statistic & $\begin{array}{c}\text { True } \\
\text { Positives }\end{array}$ & $\begin{array}{c}\text { True } \\
\text { Negatives }\end{array}$ & $\begin{array}{c}\text { False } \\
\text { Positives }\end{array}$ & $\begin{array}{c}\text { False } \\
\text { Negatives }\end{array}$ & $\begin{array}{c}\text { Matthew's Correlation } \\
\text { Coefficient (MCC) }\end{array}$ \\
\hline $\begin{array}{l}\text { Average Pairwise } \\
\text { F }_{\text {ST }} \text { (FSTA) }\end{array}$ & 66 & 0 & 100 & 34 & -0.45 \\
\hline $\begin{array}{c}\text { Range of Pairwise } \\
\text { F }_{\mathrm{ST}} \text { (FSTR) }\end{array}$ & 81 & 0 & 100 & 19 & -0.32 \\
\hline $\begin{array}{c}\mathrm{F}_{\mathrm{ST}} \text { Outlier Test } \\
\text { (PFST) }\end{array}$ & 3 & 88 & 12 & 97 & -0.17 \\
\hline $\begin{array}{l}\text { Kuhner-Felsenstein } \\
\text { Distance (KF) }\end{array}$ & 100 & 0 & 100 & 0 & 0.00 \\
\hline $\begin{array}{l}\text { Robinson-Foulds } \\
\text { Distance (RF) }\end{array}$ & 0 & 100 & 0 & 100 & 0.00 \\
\hline $\begin{array}{l}\text { Mean of Maximum } \\
\text { Likelihood (MLM) }\end{array}$ & 84 & 0 & 100 & 16 & -0.29 \\
\hline $\begin{array}{l}\text { Standard Deviation } \\
\text { of Maximum } \\
\text { Likelihood } \\
\text { (MLSD) }\end{array}$ & 71 & 95 & 5 & 29 & 0.68 \\
\hline
\end{tabular}

